\title{
Recent Trends in the Incidence and Survival of Stage 1A Pancreatic Cancer: A Surveillance, Epidemiology, and End Results Analysis
}

\author{
Amanda L. Blackford (D), ${ }^{1}$ Marcia Irene Canto, ${ }^{1,2}$ Alison P. Klein, ${ }^{1,3}$ Ralph H. Hruban, ${ }^{1,3}$ Michael Goggins (D) ${ }^{1,2,3, *}$ \\ Affiliations of authors: ${ }^{1}$ Department of Oncology, The Sol Goldman Pancreatic Cancer Research Center, Johns Hopkins Medical Institutions, Baltimore, MD, USA; \\ ${ }^{2}$ Department of Medicine, The Sol Goldman Pancreatic Cancer Research Center, Johns Hopkins Medical Institutions, Baltimore, MD, USA and ${ }^{3}$ Departments of \\ Pathology, The Sol Goldman Pancreatic Cancer Research Center, Johns Hopkins Medical Institutions, Baltimore, MD, USA \\ *Correspondence to: Michael Goggins, MD, The Sol Goldman Pancreatic Cancer Research Center, Johns Hopkins Medical Institutions, CRB2 351, 1550 Orleans Street, \\ Baltimore, MD 21231, USA (e-mail: mgoggins@jhmi.edu).
}

\begin{abstract}
Background: Rapid access to pancreatic imaging and regular pancreatic surveillance may help identify stage I pancreatic cancer. We investigated recent trends in the stage of newly diagnosed pancreatic ductal adenocarcinoma (PDACs), age at diagnosis, and survival. Methods: Trends in age-adjusted incidence of stage IA PDAC between 2004 and 2016 were determined from the National Cancer Institute's Surveillance, Epidemiology and End Results database. All tests were two-sided. Results: The incidence of stage IA PDAC cases diagnosed increased statistically significantly from 2004 to 2016 (annual percent change $=14.5,95 \%$ confidence interval $[\mathrm{CI}]=11.4$ to $17.7 ; \mathrm{P}<.001)$. During the study period, average age at diagnosis for stage IA and IB casesAQ3 declined by 3.5 years $(95 \% \mathrm{CI}=1.2$ to $5.9 ; \mathrm{P}=.004)$ and 5.5 years $(95 \% \mathrm{CI}=3.4$ to $7.6 ; \mathrm{P}<.001)$, whereas average age increased for higher-stage cases (by 0.6 to 1.4 years). Among stage IA cases, the proportion of blacks was smaller (10.2\% vs $12.5 \%)$, and the proportion of other non-Caucasians was higher compared with higher-stage cases $(11.9 \%$ vs $8.4 \% ; P<.001)$. Stage IA cases were more likely to carry insurance (vs Medicaid or none) than higher-stage cases (cases aged younger than 65 years; odds ratio $=2.45,95 \% \mathrm{CI}=1.96$ to $3.06 ; \mathrm{P}<.001)$. The 5 -year overall survival for stage IA PDAC improved from $44.7 \%(95 \%$ $\mathrm{CI}=31.4$ to 63.7$)$ in 2004 to $83.7 \%(95 \% \mathrm{CI}=78.6 \%$ to $89.2 \%)$ in 2012 ; 10 -year survival improved from $36.7 \%$ (95\% CI=24.1 to 55.8$)$ in 2004 to $49.0 \%$ (95\% CI $=37.2 \%$ to $64.6 \%$ ) in 2007. Conclusions: In recent years, the proportion of patients diagnosed with stage IA PDAC has increased, their average age at diagnosis has decreased, and their overall survival has improved. These trends may be the result of improved early diagnosis and early detection.
\end{abstract}

Pancreatic ductal adenocarcinoma (PDAC) has very poor survival, but 5-year survival rates have improved recently (currently approximately $9 \%)(1,2)$. The reasons for this improved survival are uncertain. Most patients with PDAC develop symptoms after the cancer has spread beyond the gland $(3,4)$. Patients diagnosed with stage I PDAC while undergoing pancreatic surveillance with imaging are usually asymptomatic (5). Opportunities to diagnose an asymptomatic stage I PDAC occur when patients undertake regular pancreatic surveillance either for their familial and/or genetic susceptibility (6) or for incidentally detected pancreatic cyst(s). Asymptomatic stage I PDACs can be detected incidentally when individuals undergo abdominal imaging for other indications. The risk of developing pancreatic cancer is generally highest among carriers of pathogenic pancreatic cancer susceptibility gene variants and in those with a significant family history of PDAC $(7,8)$ and intermediate in those with incidentally detected pancreatic cysts (9) and other risk factors, such as smoking (average approximately twofold elevated risk), obesity or metabolic syndrome (approximately 1.5 -fold elevated) $(10,11)$, and increasing age (approximately tenfold increase between ages 40 and 80 years) (12). The magnitude of risk depends on many factors, including the extent of PDAC family history (eg, having two first-degree relatives with PDAC confers approximately sixfold elevated risk) (13), which pathogenic susceptibility gene and variant is affected (if any), and the number of pack-years smoked (14).

Most incidentally detected neoplastic pancreatic cysts are intraductal papillary mucinous neoplasms (IPMNs), and most of these have very low risk of progressing to invasive carcinoma $(15,16)$. Pancreatic cysts are commonly found during abdominal imaging for nonpancreatic indications, with an incidence that increases with age [approximately $10 \%$ by age 70 years $(9,17,18)]$. Invasive carcinomas diagnosed in association with IPMNs can be usual PDACs or colloid carcinomas, which are often lowstage with better outcome (19). 
PDACs are not commonly detected at stage IA in the absence of regular surveillance because they usually do not cause symptoms and may progress relatively quickly to higher stages (20). Regular pancreatic surveillance of individuals with familial/ genetic susceptibility has been reported to result in the downstaging of PDAC, with improved 5-year survival compared with PDAC diagnosed after symptomatic presentation $(5,21)$. Median 5-year survival for patients with localized PDAC who undergo pancreatic resection and (neo)-adjuvant chemotherapy is approximately $20 \%$ and higher for stage I cases (22-25). With the emergence of pancreatic surveillance programs for individuals with familial/genetic risk, and improvements in the detection and management of pancreatic cystic neoplasms, we suspected better early detection could be contributing to the improvement in PDAC survival. If so, this would be reflected in a downward stage shift, with an increase in the incidence of stage IA PDAC being diagnosed. We analyzed data from the Surveillance, Epidemiology and End Results (SEER) registry, representative of the US population, to assess recent trends in the stage of newly diagnosed PDACs, age at diagnosis, and survival.

\section{Methods}

\section{Data Source and Study Patients}

Individuals included in the SEER registries diagnosed with PDAC between January 1, 2004, and December 31, 2016, were identified using the SEER Program (www.seer.cancer.gov). Most PDACs, including small-sized tumors, have lymph node involvement at diagnosis; imaging often misses nodal involvement and therefore understages $(26,27)$. Thus, most PDACs classified by imaging alone as stage I would be restaged as stage IIB or stage III after surgical pathology. Indeed, PDAC cases coded as stage IA nonsurgical in SEER are older and their overall survival approximates that of stage IIB surgical cases (Table 1; Supplementary Table 1, Supplementary Figures 1-3, available online). Therefore, we only included stage I or II cases in analyses if they had surgery documented by SEER. Insurance status was grouped as none, Medicaid, or any other insurance (private or Medicare), and analyzed separately by age dichotomized at 65 years, because cases older than 65 years classified by SEER as uninsured are likely Medicare eligible.

\section{Statistical Analysis}

Cohort demographic and clinical characteristics were summarized and compared between patients with and without stage IA cancers using $t$ tests, Wilcoxon rank-sum, and Fisher exact tests with $P$ values calculated using Monte Carlo simulation. Age-adjusted pancreas cancer incidence rates and corresponding annual percent changes (APC) were calculated using SEER*stat (v8.3.5) and Joinpoint software (v4.7.0), respectively. Incidence rates were standardized to the 2000 US population and calculated separately by stage and other subgroups within stage IA (race, age, IPMN-related disease, geographic region) $(28,29)$. All tests were two-sided, and a $P$ value of less than .05 was considered statistically significant.

Linear regression was used to describe changes in age and tumor size at diagnosis over time, with differences in trajectories across stage groups assessed with interaction analysis. Cox proportional hazards models were used to test for linear trends in survival according to year of diagnosis, adjusting for relevant variables (see also Supplementary Methods, available online).
Table 1. Characteristics of cases in SEER registries diagnosed with pancreatic ductal adenocarcinoma from 2004 to 2016

\begin{tabular}{|c|c|c|}
\hline Characteristic* $^{*}$ & $\begin{array}{l}\text { All other staged } \\
\text { cancers }(\mathrm{n}=91478)\end{array}$ & $\begin{array}{l}\text { Stage 1A PDAC } \\
\quad(\mathrm{n}=1719)\end{array}$ \\
\hline Age at diagnosis, mean (SD), y & $67.5(12.3)$ & $61.4(13.0)$ \\
\hline \multicolumn{3}{|l|}{ Race, No. (\%) } \\
\hline White & $72381(79.2)$ & $1329(77.9)$ \\
\hline Black & $11392(12.5)$ & $174(10.2)$ \\
\hline Other & $7637(8.4)$ & $203(11.9)$ \\
\hline Unknown & 68 & 13 \\
\hline \multicolumn{3}{|l|}{ Sex, No. (\%) } \\
\hline Male & $46988(51.4)$ & $814(47.4)$ \\
\hline Female & $44490(48.6)$ & $905(52.6)$ \\
\hline Hispanic ethnicity, No. (\%) & 9927 (10.9) & $186(10.8)$ \\
\hline \multicolumn{3}{|l|}{ SEER Region, No. (\%) } \\
\hline Pacific Northwest & $46581(50.9)$ & $930(54.1)$ \\
\hline Midwest & $9284(10.1)$ & $132(7.7)$ \\
\hline Southeast & $19821(21.7)$ & $348(20.2)$ \\
\hline Northeast & $15792(17.3)$ & $309(18)$ \\
\hline Married, Yes/No (\%) & $50248 / 87854$ (57.2) & $1103 / 1638(67.3)$ \\
\hline \multicolumn{3}{|l|}{ Location, No. (\%) } \\
\hline Urban & $80993(88.7)$ & $1574(91.7)$ \\
\hline Metro & $9126(10)$ & $125(7.3)$ \\
\hline Rural & $1237(1.4)$ & $18(1)$ \\
\hline Unknown & 122 & 2 \\
\hline \multicolumn{3}{|l|}{$\begin{array}{l}\text { Year of Diagnosis, } \\
\text { No. (\% of total per year) }\end{array}$} \\
\hline 2004 & $5581(99.3)$ & $38(0.07)$ \\
\hline 2005 & $5760(99.0)$ & $56(1.0)$ \\
\hline 2006 & $5976(99.1)$ & $53(0.09)$ \\
\hline 2007 & $6198(99.1)$ & $59(0.09)$ \\
\hline 2008 & $6503(98.9)$ & $74(1.1)$ \\
\hline 2009 & $6737(98.7)$ & $91(1.3)$ \\
\hline 2010 & $7182(98.5)$ & $113(1.5)$ \\
\hline 2011 & 7265 (98.2) & $132(1.8)$ \\
\hline 2012 & $7529(97.5)$ & $196(2.5)$ \\
\hline 2013 & $7877(97.4)$ & $214(2.6)$ \\
\hline 2014 & $8125(97.5)$ & $206(2.5)$ \\
\hline 2015 & $8347(97.0)$ & $255(3.0)$ \\
\hline 2016 & $8398(97.3)$ & $232(2.7)$ \\
\hline Tumor size, median (range) & $3.9(0.1-10)$ & $1.5(0.1-2.5)$ \\
\hline \multicolumn{3}{|l|}{ Insurance status, No. (\%) } \\
\hline None & $2222(3)$ & $25(1.6)$ \\
\hline Medicaid & $9298(12.5)$ & $118(7.5)$ \\
\hline Other (private or Medicare) & $62641(84.5)$ & 1429 (90.9) \\
\hline Unknown $^{\dagger}$ & 17317 & 147 \\
\hline \multicolumn{3}{|l|}{$\begin{array}{l}\text { IPMN-associated disease, No. } \\
\text { (\%) }\end{array}$} \\
\hline No & $88301(96.5)$ & $1595(92.8)$ \\
\hline Yes & $3177(3.5)$ & $124(7.2)$ \\
\hline Received surgery, No. (\%) & $18305(20)$ & $1719(100)$ \\
\hline $\begin{array}{l}\text { Received neoadjuvant radia- } \\
\text { tion, Yes/No (\%) }\end{array}$ & $858 / 18008(4.8)$ & 19/1714 (1.1) \\
\hline \multicolumn{3}{|l|}{ Tumor location } \\
\hline Head & $43696(47.8)$ & $630(36.6)$ \\
\hline Body & 11791 (12.9) & $316(18.4)$ \\
\hline Tail & $13717(15)$ & $491(28.6)$ \\
\hline Other location & $22274(24.3)$ & $282(16.4)$ \\
\hline
\end{tabular}

${ }^{*} \mathrm{P}$ values for Fisher exact test for categorical variables and for $t$ tests for continuously measured variables for differences in variables between cases with Stage IA disease and all other staged cases were $<.001$ for all variables except for Hispanic ethnicity $(\mathrm{p}=.97)$. IPMN $=$ intraductal papillary mucinous neoplasms; PDAC = pancreatic ductal adenocarcinoma; SEER = Surveillance, Epidemiology, and End Results.

†Insurance status not available for cases diagnosed in 2004-2006. 


\section{Results}

\section{Baseline Characteristics}

Demographic and clinical characteristics of stage IA vs all other PDACs diagnosed from 2004 to 2016 in SEER are shown in Table 1 and Supplementary Table 1 (available online). Only 1.1\% of stage IA cases that underwent surgery received neoadjuvant

Table 2. Odds ratios carrying insurance (Medicare or private) compared with Medicaid or no insurance according to stage of disease, separately for those aged younger than 65 years or 65 years and older at PDAC diagnosis

\begin{tabular}{lll}
\hline Stage of disease & \multicolumn{1}{c}{ OR (95\% CI) } & $P^{*}$ \\
\hline Age $<65 \mathrm{y}$ & & \\
Stage IA & 1.00 (referent) & .003 \\
Stage IB & 0.66 (0.50 to 0.87$)$ & $<.001$ \\
Stage IIA & $0.44(0.34$ to 0.56$)$ & $<.001$ \\
Stage IIB & $0.55(0.44$ to 0.70$)$ & $<.001$ \\
Stage III & $0.42(0.33$ to 0.53$)$ & $<.001$ \\
Stage IV & 0.36 (0.29 to 0.45$)$ & \\
Age $\geq 65$ y & & .13 \\
Stage IA & 1.00 (referent) & .002 \\
Stage IB & $0.71(0.45$ to 1.10$)$ & .08 \\
Stage IIA & $0.56(0.39$ to 0.81$)$ & .02 \\
Stage IIB & $0.72(0.50$ to 1.03$)$ & $<.001$ \\
Stage III & $0.65(0.45$ to 0.94$)$ & \\
Stage IV & 0.53 (0.37 to 0.75$)$ & \\
\hline
\end{tabular}

*Estimates and two-sided $P$ values are calculated from logistic regression models for carrying Medicare or private insurance compared with Medicaid or no insurance according to stage of disease and are adjusted for age, sex, race, marital status, SEER region, Hispanic ethnicity, and tumor location. $\mathrm{CI}=$ confidence interval; OR = odds ratio; PDAC = pancreatic ductal adenocarcinoma; SEER = Surveillance, Epidemiology, and End Results. therapy during the study period; this percentage has increased considerably in recent years. Among all cases, those diagnosed with stage IA PDAC were approximately 6 years younger on average $(61.4$ vs 67.5 years; $P<.001)$ and more were female $(52.6 \%$ vs $48.6 \% ; \quad P<.001)$ than those with higher-stage cancers. Compared to higher-stage cases, stage IA cases were more likely to be married ( $67.3 \%$ vs $57.2 \%$ ), less likely to be black $(10.2 \%$ vs $12.5)$, more likely to be of other races ( $11.9 \%$ vs $8.4 \%$ ), and more likely to reside in an urban over a metro or rural area (91.7\% vs 88.7\%) (all $P<.001$, Fisher test). Stage IA cases aged younger than 65 years were also more likely than higher-staged cases to have insurance other than Medicaid, after adjusting for age, race, marital status, Hispanic ethnicity, sex, SEER region, and tumor location (odds ratio $[\mathrm{OR}]=2.45,95 \% \mathrm{CI}=1.96$ to 3.06 ; $P<.001)$. A similar result was observed for cases age older than 65 years $(\mathrm{OR}=1.77,95 \% \mathrm{CI}=1.24$ to $2.51 ; \mathrm{P}=.002$; Tables 1 ; and 2 Supplementary Table 2, available online).

\section{Incidence Trends}

The incidence of all PDAC cases increased over the study period (APC $=1.4,95 \% \mathrm{CI}=1.2$ to $1.6 ; \mathrm{P}<.001$ ), but the corresponding increase in stage IA cases over the same period was tenfold higher $(\mathrm{APC}=14.5,95 \% \mathrm{CI}=11.4$ to $17.7 ; \mathrm{P}<.001$; Figure 1 ).

We further examined the trends in each stage group (Figure 1). The incidence of stage IV PDAC increased the smallest over the study period ( $\mathrm{APC}=0.57,95 \% \mathrm{CI}=0.35$ to 0.79 ; $\mathrm{P}<.001$ ), followed by stage III (APC $=1.4,95 \% \mathrm{CI}=0.79$ to 2.1 ; $\mathrm{P}=.001$ ) and stage IIA (APC $=2.9,95 \% \mathrm{CI}=2.0$ to $3.9 ; \mathrm{P}<.001$ ). Stage IB disease increased from 2004 to 2011 (APC $=3.6,95 \%$ CI $=1.4$ to $5.9 ; \mathrm{P}=.005$ ) with greater increases from 2011 to 2016 $(\mathrm{APC}=8.3,95 \% \mathrm{CI}=5.3$ to $11.4 ; \mathrm{P}<.001)$. In contrast, stage IIB disease increased from 2004 to 2009 (APC $=6.9,95 \% \mathrm{CI}=3.8$ to 10.1 ;

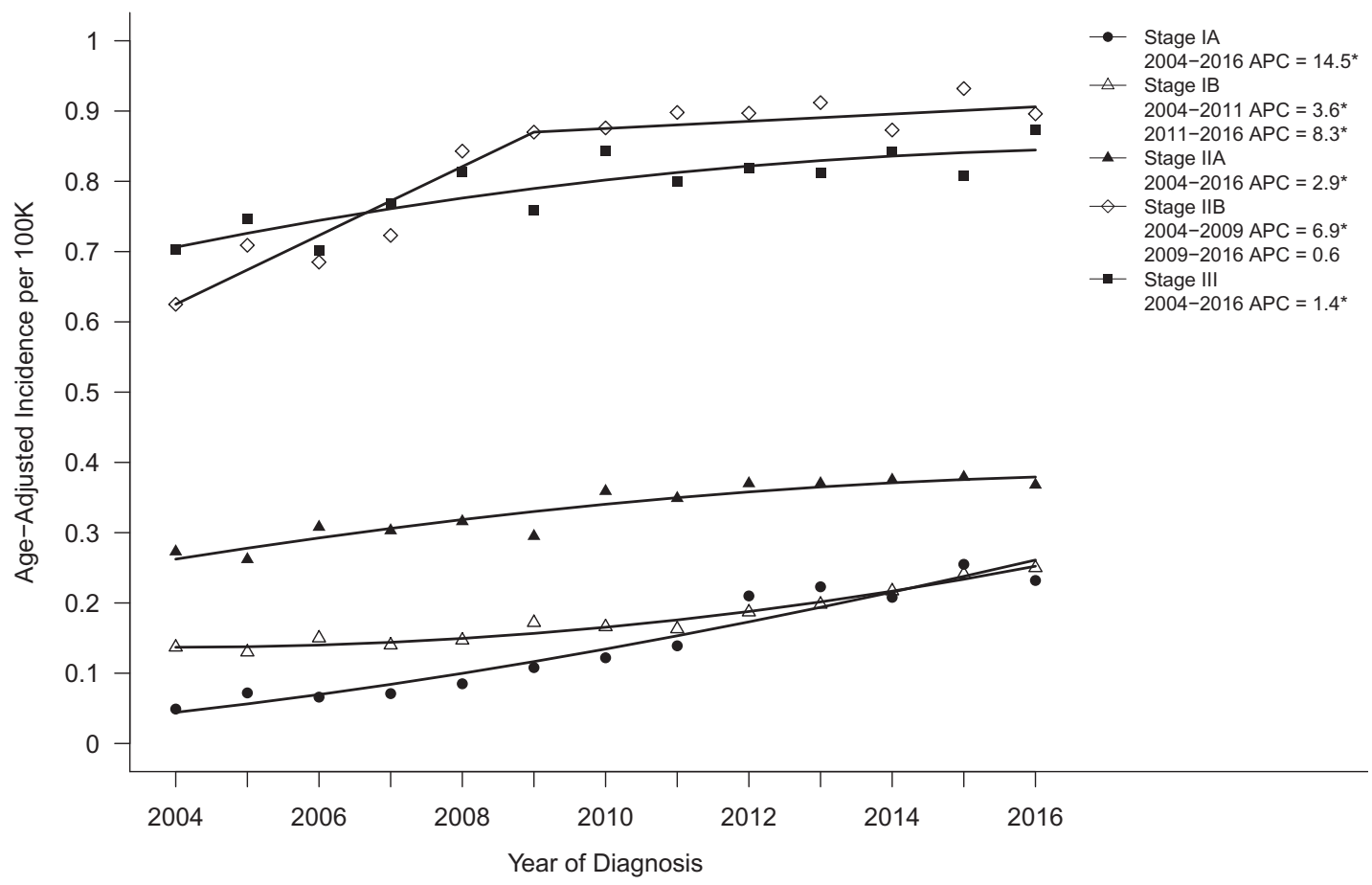

Figure 1. Age-adjusted incidence trends over time for pancreatic cancer stage IA-III, shown as age-adjusted incidence rates (2004-2016). Asterisks denote annual percent change (APC) with $\mathrm{P}<.05$. 
A

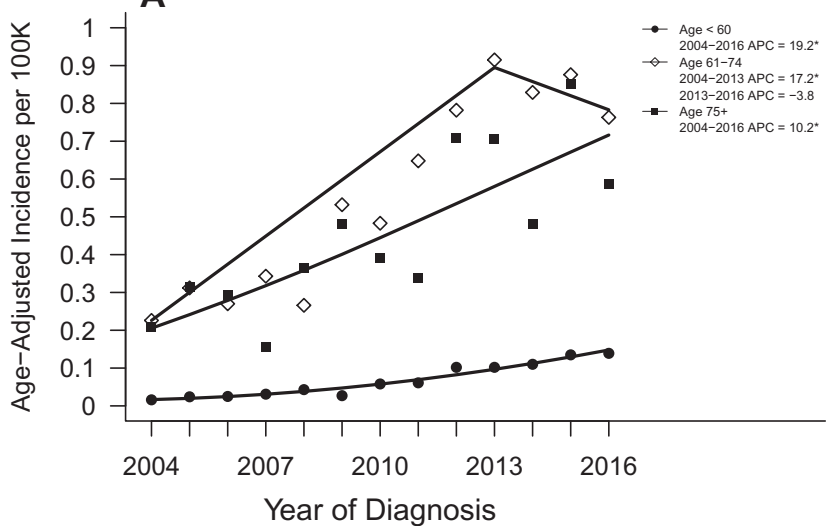

C

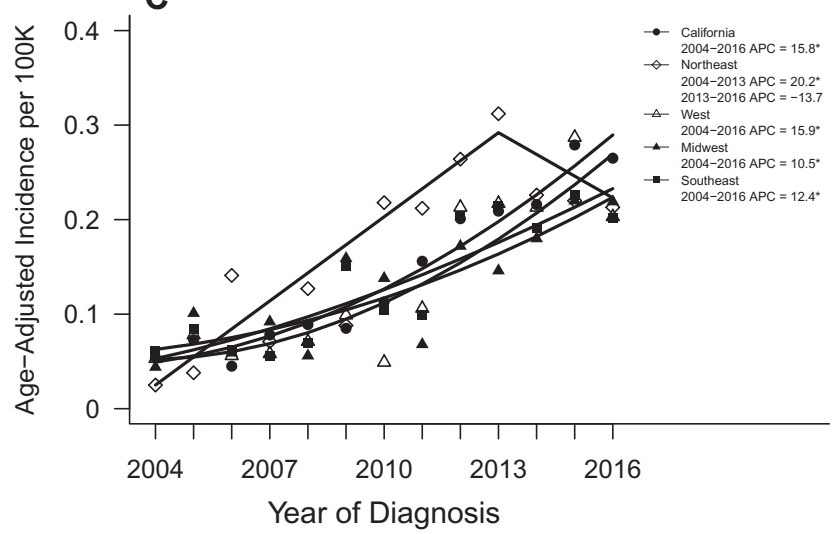

B

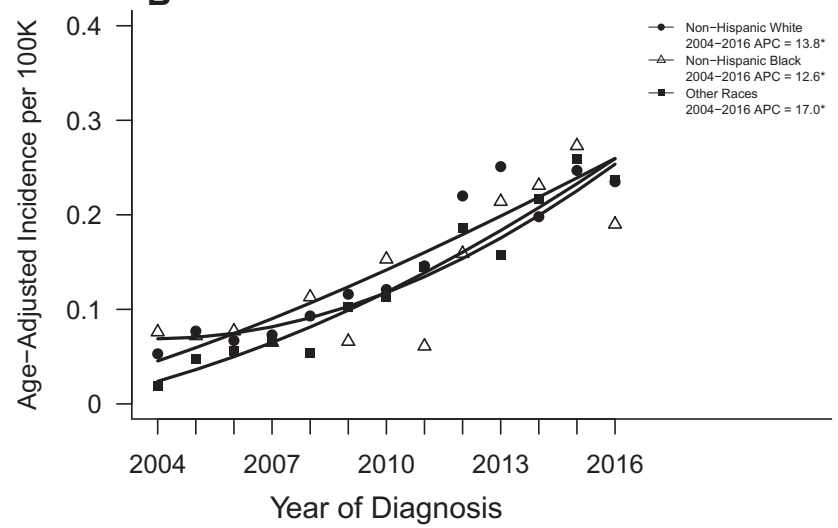

D

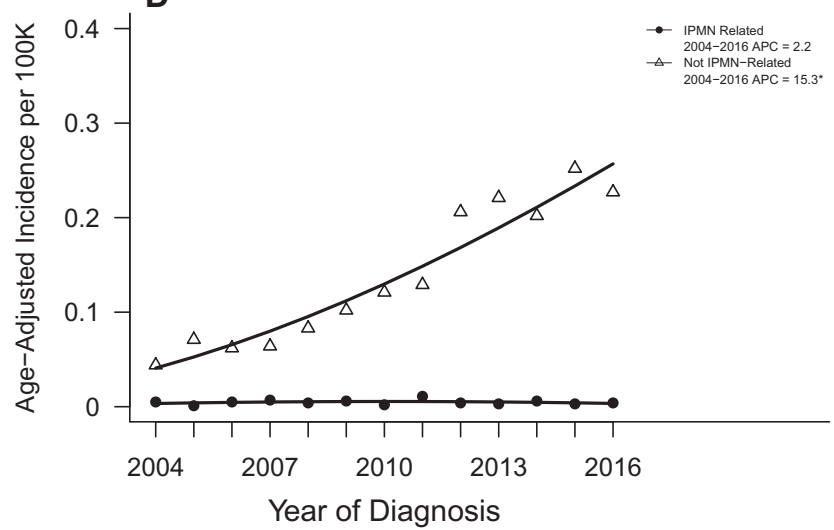

Figure 2. Age-adjusted incidence trends over time for pancreatic cancer stage IA within relevant subgroups. Trends shown according to (A) age, (B) race, (C) geographic SEER region, and (D) IPMN-related disease status. Asterisks denote annual percent change (APC) with two-sided $P<.05$. IPMN $=$ intraductal papillary mucinous neoplasms.

$P<.001$ ) but leveled off from 2010 to 2016 (APC $=0.6,95 \% \mathrm{CI}=-0.86$ to $2.1 ; \mathrm{P}=.37$ ).

The trend in stage IA PDAC diagnoses was most evident in younger (younger than 60 years) compared to older cases (either 61-74 years or older than 75 years) at diagnosis. Whereas the incidence of stage IA diagnoses increased statistically significantly for each age group during the study period, the increase was larger for those younger than 60 years (APC $=19.2,95 \% \mathrm{CI}=$ 15.4 to $23.1 ; P<.001)$ compared with those aged $61-74$ years, where incidence increased from 2004 to 2013 (APC $=17.2,95 \%$ $\mathrm{CI}=11.7$ to $23.1 ; \mathrm{P}<.001$ ) but leveled off from 2013 to 2016 (APC $=-3.8,95 \% \mathrm{CI}=-13.4$ to $18.3 ; \mathrm{P}=.60)$, and those aged older than 75 years $(\mathrm{APC}=10.2,95 \% \mathrm{CI}=5.1$ to $15.6 ; P=.001$, Figure 2 ).

The incidence of stage IA diagnoses increased statistically significantly over time in whites, blacks, and other races (APC = 13.8, 12.6, 17.0, respectively; $P<.001$ for each). The increase in stage IA diagnoses was observed in all SEER regions. This increase was not as large in the Southeast (APC $=12.4,95 \% \mathrm{CI}=$ 7.5 to $17.5 ; P<.001)$ or the Midwest $(\mathrm{APC}=10.5,95 \% \mathrm{CI}=5.4$ to 15.9; $\mathrm{P}<.001$ ) compared with California (APC $=15.8,95 \% \mathrm{CI}=$ 12.9 to $18.9 ; P<.001$ ) or the West $(\mathrm{APC}=15.9,95 \% \mathrm{CI}=9.9$ to 22.2; $P<.001)$. In the Northeast, incidence increased significantly from 2004 to 2013 ( $\mathrm{APC}=20.2,95 \% \mathrm{CI}=8.0$ to 33.8 ; $\mathrm{P}=.004$ ) but decreased from 2013 to 2016 ( $\mathrm{APC}=-13.7,95 \% \mathrm{CI}=$ -41.2 to $26.6 ; P=.40$; Figure 2 )

To examine the extent to which trends in stage IA PDACs might be related to an increase in stage IA IPMN-associated PDACs, as a result of surveillance of patients with IPMN, we examined trends in this diagnosis (Table 1). Overall, 3301 (3.5\%) of the 93197 PDAC cases received an IPMN-associated PDAC diagnosis. Within stage IA, $7.2 \%$ of cases were IPMN-associated, compared with $3.5 \%$ for all other stages $(\mathrm{OR}=2.13,95 \% \mathrm{CI}=1.75$ to 2.58 , in which a stage IA case was IPMN-associated; $P<.001$ ). The incidence of stage IA PDACs not IPMN-associated increased over the study period $(\mathrm{APC}=15.3,95 \% \mathrm{CI}=11.5$ to $19.3 ; \mathrm{P}<.001)$ without any statistically significant trend in the incidence of IPMN-associated stage IA (APC $=2.2,95 \% \mathrm{CI}=-6.2$ to 11.3 ; $\mathrm{P}=$.59; Figure 2).

Consistent with the increase in age-specific incidence of stages IA, IB, and, to a lesser extent IIA, PDAC cases (Figure 1; Supplementary Figure 2, available online), there was an increase in the incidence of patients who underwent surgical resection $(\mathrm{APC}=2.8,95 \% \mathrm{CI}=2.3$ to $3.3 ; \mathrm{P}<.001)$ with a relatively small change in the incidence of cases without a surgical pathologic diagnosis ( $\mathrm{APC}=0.26,95 \% \mathrm{CI}=0.06$ to $0.46 ; \mathrm{P}=.01$ ) and a nonsignificant decrease in cases staged as stage II not otherwise specified ( $\mathrm{APC}=-1.6,95 \% \mathrm{CI}=-6.2$ to $3.2 ; \mathrm{P}=.47)$. There was an increase from 2004 to 2013 ( $\mathrm{APC}=4.0,95 \% \mathrm{CI}=3.4$ to 4.6 ; $P<.001)$ in the incidence of cases with localized disease but did not have surgery, but this trend dropped off from 2013 to 2016 $(\mathrm{APC}=-1.9,95 \% \mathrm{CI}=-4.5$ to $0.89 ; \mathrm{P}=.16)$. Whereas the incidence of surgical cases increased (APC $=2.8$ ), the incidence of stage IA cases increased (APC $=14.5)$, indicating the overall increase in surgeries performed for patients with PDAC was not the main explanation for the increase in stage IA cases diagnosed. 


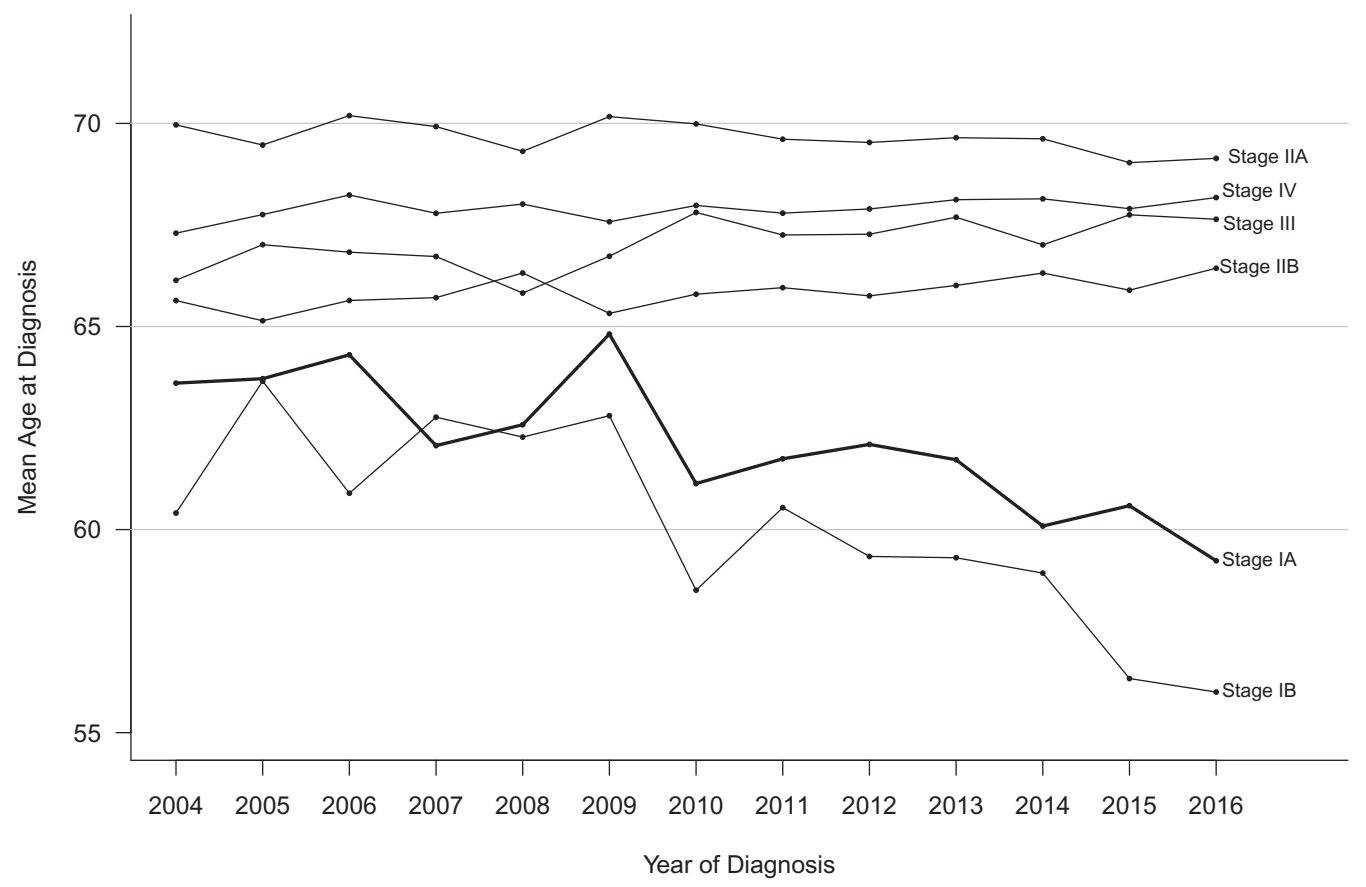

Figure 3. Trends in 5-year overall survival probabilities according to pancreatic cancer stage (2004-2012).

Average tumor size at diagnosis for stage IA cases decreased slightly $(0.009 \mathrm{~cm} /$ year, $95 \%$ CI $=-0.017$ to $-0.006 ; \quad P=.04$; Supplementary Figure 4, available online); small tumor size reductions were also noted for stages IB and IIA PDACs but were relatively constant for stages IIB, III, and IV during the study period.

\section{Age at Diagnosis Trends}

We next calculated the mean age at PDAC diagnosis yearly for 2004 to 2016 by stage (Figure 3). Over the 13-year study period, average age at diagnosis for stage IA cases declined by 3.5 years (95\% CI $=1.2$ to $5.9 ; P=.004)$. Cases diagnosed with stage IB PDAC demonstrated an even larger decline in age at diagnosis (5.5 years, $95 \% \mathrm{CI}=3.4$ to $7.6 ; \mathrm{P}<.001$ ). In contrast, mean age at diagnosis for stage IIA cases remained unchanged, and the other stages (IIB, III, and IV) demonstrated small increases (0.6-1.4 years over the 13-year period). Trajectories for mean age over the period for stages IIA, IIB, III, and IV were all statistically significantly different than the stage IA trajectory (interaction $P<.001,5 \mathrm{df}$ ).

\section{Trends in Survival}

Overall survival after a PDAC diagnosis improved from 2004 to 2016 for all TNM stages. Stage IA cases demonstrated the largest reduction in mortality per continuously increasing year of diagnosis $(\mathrm{HR}=0.93,95 \% \mathrm{CI}=0.87$ to 0.98$)$, with stages IB and III cases following a similar pattern $(\mathrm{HR}=0.94,95 \% \mathrm{CI}=0.90$ to 0.98 , and $\mathrm{HR}=0.95,95 \% \mathrm{CI}=0.94$ to 0.96 ; respectively); hazard ratios for all other cases by stage ranged from 0.97 to 0.98 (interaction $P<.001$ with $5 \mathrm{df}$ ). Specifically, 5-year overall survival for stage IA cases improved from $44.7 \%(95 \% \mathrm{CI}=34.1 \%$ to $63.7 \%)$ in 2004 to $83.7 \%$ (95\% CI $=78.6 \%$ to $89.2 \%$ ) in 2012 (Figure 4). Large improvements in 5-year survival were also observed for stage IB cases $(52.6 \%, 95 \% \mathrm{CI}=44.0 \%$ to $63.0 \%$, to $74.3 \%, 95 \% \mathrm{CI}=68.0 \%$ to $81.3 \%$, and more modest improvements were observed for higher stage cases: stage IIA $(8.2 \%, 95 \% \mathrm{CI}=6.3 \%$ to $10.6 \%$, to $13.3 \%, 95 \% \mathrm{CI}=11.0 \%$ to $16.1 \%)$; stage IIB $(10.8 \%, 95 \% \mathrm{CI}=8.7 \%$ to $13.4 \%$, to $15.5 \%, 95 \% \mathrm{CI}=13.2 \%$ to $18.1 \%)$; stage III $(2.5 \%, 95 \%$ $\mathrm{CI}=1.5 \%$ to $4.2 \%$, to $3.2 \%, 95 \% \mathrm{CI}=2.1 \%$ to $4.9 \%$; ; and stage $\mathrm{IV}$ ( $0.9 \%, 95 \% \mathrm{CI}=0.6 \%$ to $1.3 \%$, to $2.8 \%, 95 \% \mathrm{CI}=2.4 \%$ to $3.4 \%)$ (Figure 4). Among stage IA cases, improvements in survival were similar across groups defined by age, race, IPMN-related disease, and geographic region (interaction $P$ values for group by year of diagnosis >0.05; Supplementary Figure 5, available online). The improved survival in the whole stage IA cohort continued with longer follow-up: 10-year survival for stage IA cases improved from $36.7 \%$ ( $95 \% \mathrm{CI}=24.1 \%$ to $55.8 \%$ ) to $49.0 \%$ (95\% CI $=37.2 \%$ to $64.6 \%$ ) for cases diagnosed in 2004 and 2007, respectively.

\section{Discussion}

We find that the proportion of patients diagnosed with stage IA PDAC has increased statistically significantly and the average age at diagnosis of these patients has decreased in recent years. It is unlikely that this trend is the result of changes in disease presentation; patients who present with symptoms generally have advanced-stage disease. Nor is it likely to be related to changes in ascertainment by SEER, because any such change would not be expected to result in a selective decrease in the average age of patients with a stage IA diagnosis. Instead, we suspect the increased diagnoses of stage IA pancreatic cancers is the result of several factors, including earlier diagnosis and surgical management, improved insurance coverage, and enrollment of more eligible individuals (both those with incidentally detected pancreatic cysts and those with familial and/or genetic risk) into pancreatic surveillance programs.

We cannot rule out other explanations such as changes in the biology of the disease, but the disproportionate increase in stage IA cases has occurred over a short period of time, making biological factors less likely. The average age of patients at 


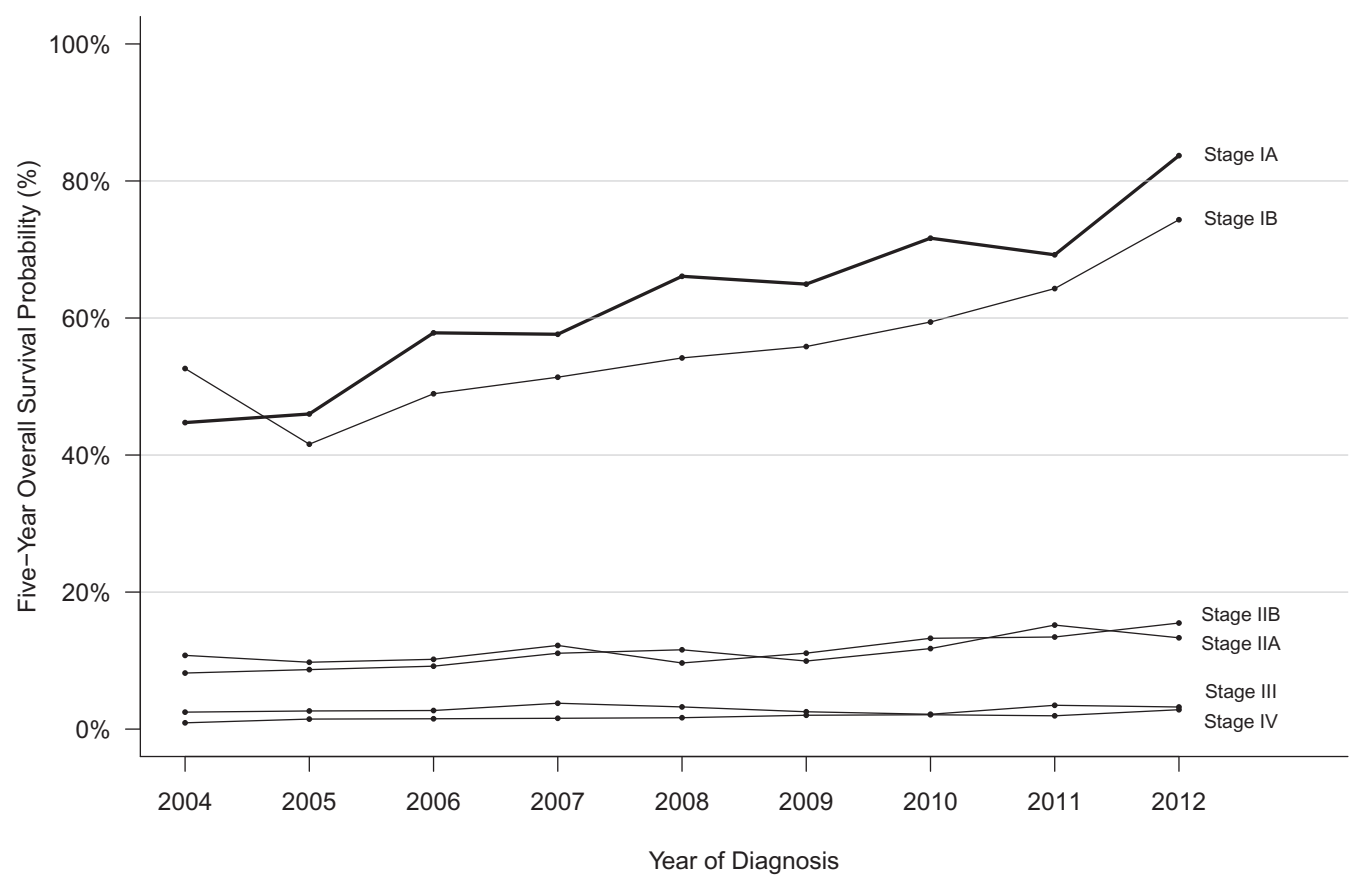

Figure 4. Trends in mean age at diagnosis according to pancreatic cancer stage (2004-2016).

diagnosis with stage IA disease decreased statistically significantly over the study period with a similar decrease in age for stage IB cases, but there was no downward trend in age overall-a trend that might represent a cohort effect. Such a cohort effect has been observed recently for young-onset colorectal cancer (30). Instead, the increasing numbers of stage IA cases were most notable in younger individuals, which may in part reflect the emergence of pancreatic surveillance of individuals with familial/genetic risk and surveillance of patients with IPMN. Individuals with inherited gene mutations that predispose to PDAC are diagnosed with the disease at younger ages (by approximately 3-5years on average) than individuals with sporadic forms of PDAC (31-33) and are generally recommended to begin pancreatic surveillance at age 50 (mutation carriers) or 55 (for familial risk) years (5). IPMN-associated PDAC also occurs in younger individuals (34), and prior studies have found IPMNassociated PDACs are more likely to be diagnosed at lower stage (19). There was also an increase in the proportion of IPMNassociated stage IA PDACs during the study period, but less than $5 \%$ of all stage IA PDACs in SEER were IPMN-associated. Overall, $3.4 \%$ of all PDACs during the study period were classified with IPMN-associated codes; somewhat less than the $6 \%$ reported in a hospital series of more than 2500 pancreatic resections (35). The percentage of IPMN-associated PDACs likely underestimates the value of pancreatic surveillance for patients with IPMN because pancreatic resection of IPMN 1) may prevent pancreatic cancer development $(5,15,16)$ and 2) may identify early pancreatic cancer elsewhere in the gland not arising from their IPMN.

Other factors that may have contributed to the trends in stage IA PDACs diagnosed during the study period include better diagnostic imaging and increased suspicion of pancreatic cancer as a diagnosis leading to better pursuit of subtle symptoms or subtle pancreatic imaging abnormalities (36). These factors and perhaps better surgical selection (37) led to an approximate $25 \%$ increase in the incidence of PDAC cases that underwent surgery, which by itself would be expected to yield a similar increase in the proportion of stage IA cases. An emerging factor is increased recognition that new-onset diabetes in an older person could be a presentation of pancreatic cancer (38). Trends in the numbers of abdominal scans performed are not a likely explanation for the trend in stage IA PDAC diagnoses. If the stage IA trends were primarily related to incidental detection, abdominal imaging scans performed during the study period would have had to increase by $14 \%$ annually to explain this increase. Instead, recent trends suggest modest declines in the numbers of abdominal computed tomography scans being performed (39-41).

We found having insurance, either private or Medicare, was associated with greater odds of being diagnosed with a stage IA PDAC compared with those on Medicaid or without insurance, independent of race, ethnicity, marital status, and other factors, supporting the hypothesis that underinsurance and/or poverty, lower health-care access, and utilization reduces the likelihood of having the earliest-stage and most-curable PDAC. Access to care and health-care utilization are likely important determinants of having a stage IA PDAC. One indicator of health-care utilization is marital status, because health-care utilization is higher among married individuals (42), and stage IA cases were more likely to be married (Table 1). Smokers, who are more likely to be under- or uninsured (43), are diagnosed with PDAC at younger ages on average than nonsmokers, raising the possibility that improvement of insurance coverage could also have contributed to the younger average age at diagnosis for stages IA and IB cases (44). Regional differences in the stage IA PDAC trends are likely due to multiple factors, including availability of pancreas screening programs and population differences, but health-care coverage in a region is also likely to be an important factor. Prior studies have reported blacks more often present with higher-stage PDAC, likely because of multiple factors including insurance coverage (45-47). Our findings support other studies that show access to care and insurance status impacts 
early cancer detection. Insurance status is strongly associated with cancer outcomes, and early stage, screen-detectable cancers are more likely for patients who are insured (48). The implementation of the Affordable Care Act has steadily reduced the amount of uninsured individuals in the United States to a record low of 9\% during 2017 (48). This has led to a dramatic reduction in insurance disparity, which is thought to have contributed to an increase in the incidence of several stage I cancers including pancreatic cancer in states associated with Medicaid expansion compared with states that did not expand insurance (49). Our analysis shows the increased incidence of stage IA PDACs was evident after 2005 and continued after the implementation of the Affordable Care Act in 2010 (Figure 1).

A strength of our study is the 13-year analysis of trends in national cancer registry data. The SEER registry has some recognized limitations with respect to data accuracy and data completion including the accuracy of the original pathologic diagnosis and staging (50); SEER also lacks covariate data needed to develop more comprehensive prediction models, and as a cancer-reporting registry, SEER may underreport precursor lesions, including IPMNs, but such limitations to our study do not appear to explain the trends found for stage IA PDAC during the study period. Although the incidence and survival trends for stage IA PDAC are encouraging, stage IA PDAC still represents only a fraction (approximately $3 \%$ ) of patients at diagnosis.

In conclusion, the proportion of patients diagnosed with stage IA pancreatic cancer has been increasing in recent years, and their average age at diagnosis has decreased without any significant change in the average age at diagnosis for patients with other disease stages, trends that point to the effect of early detection. The overall survival of stage IA pancreatic cancer has been improving in recent years and is much higher than generally appreciated.

\section{Funding}

This work was supported by NIH grants (U01CA210170, CA62924, and R01CA176828, NCI P30 CA006973), and by a Stand Up To Cancer-Lustgarten Foundation Pancreatic Cancer Interception Translational Cancer Research Grant (grant no.: SU2C-AACR-DT25-17). Stand Up To Cancer is a program of the Entertainment Industry Foundation. Research grants are administered by the American Association for Cancer Research, the scientific partner of SU2C. MG is the Sol Goldman Professor of Pancreatic Cancer Research.

\section{Notes}

The authors do not have any disclosures to report. The funders had no role in the design of the study; the collection, analysis, and interpretation of the data; the writing of the manuscript; and the decision to submit the manuscript for publication.

\section{References}

1. Siegel RL, Miller KD, Jemal A. Cancer statistics, 2019. CA A Cancer J Clin. 2019; 69(1):7-34.

2. Huang L, Jansen L, Balavarca Y, et al. Stratified survival of resected and over all pancreatic cancer patients in Europe and the USA in the early twenty-firs century: a large, international population-based study. BMC Med. 2018;16(1): 125.

3. Kawada N, Uehara H, Katayama K, et al. Diagnostic clues and subsequent examinations that detected small pancreatic cancer. Hepatogastroenterology. 2012;59(118):1665-1669.
4. Gobbi PG, Bergonzi M, Comelli M, et al. The prognostic role of time to diagnosis and presenting symptoms in patients with pancreatic cancer. Cancer Epidemiol. 2013;37(2):186-190.

5. Canto MI, Almario JA, Schulick RD, et al. Risk of neoplastic progression in individuals at high risk for pancreatic cancer undergoing long-term surveillance. Gastroenterology. 2018;155(3):740-751.e2.

6. Canto MI, Harinck F, Hruban RH, et al. International consensus recommendations on the management of patients with increased risk for familial pancreatic cancer (The Cancer of the Pancreas Screening (CAPS) Consortium Summit). Gut. 2013;62(3):339-347.

7. Abe T, Blackford AL, Tamura $\mathrm{K}$, et al. Deleterious germline mutations are a risk factor for neoplastic progression among high-risk individuals undergoing pancreatic surveillance. J Clin Oncol. 2019;37(13):1070-1080.

8. Klein AP, Brune KA, Petersen GM, et al. Prospective risk of pancreatic cancer in familial pancreatic cancer kindreds. Cancer Res. 2004;64(7):2634-2638.

9. Kromrey ML, Bulow R, Hubner J, et al. Prospective study on the incidence, prevalence and 5-year pancreatic-related mortality of pancreatic cysts in a population-based study. Gut. 2018;67(1):138-145.

10. Klein AP, Lindstrom S, Mendelsohn JB, et al. An absolute risk model to identify individuals at elevated risk for pancreatic cancer in the general population. PLoS One. 2013;8(9):e72311.

11. Andersen DK, Andren-Sandberg A, Duell EJ, et al. Pancreatitis-diabetes-pancreatic cancer: summary of an NIDDK-NCI workshop. Pancreas. 2013;42(8): 1227-1237.

12. Meza R, Jeon J, Moolgavkar SH, et al. Age-specific incidence of cancer: phases, transitions, and biological implications. Proc Natl Acad Sci U S A. 2008;105(42): 16284-16289.

13. Brune KA, Lau B, Palmisano E, et al. Importance of age of onset in pancreatic cancer kindreds. J Natl Cancer Inst. 2010;102(2):119-126.

14. Wood LD, Yurgelun MB, Goggins MG. Genetics of familial and sporadic pancreatic cancer. Gastroenterology. 2019;156(7):2041-2055.

15. Crippa S, Bassi C, Salvia R, et al. Low progression of intraductal papillary mucinous neoplasms with worrisome features and high-risk stigmata undergoing non-operative management: a mid-term follow-up analysis. Gut. 2017; 66(3):495-506.

16. Pergolini I, Sahora K, Ferrone CR, et al. Long-term risk of pancreatic malignancy in patients with branch duct intraductal papillary mucinous neoplasm in a referral center. Gastroenterology. 2017;153(5):1284-1294.e1.

17. de Jong $\mathrm{K}$, Nio CY, Hermans JJ, et al. High prevalence of pancreatic cysts detected by screening magnetic resonance imaging examinations. Clin Gastroenterol Hepatol. 2010;8(9):806-811.

18. Brewer Gutierrez OI, Lennon AM. Pancreatic cysts: sinister findings or incidentalomas? Med Clin North Am. 2019;103(1):163-172.

19. Poultsides GA, Reddy S, Cameron JL, et al. Histopathologic basis for the favorable survival after resection of intraductal papillary mucinous neoplasmassociated invasive adenocarcinoma of the pancreas. Ann Surg. 2010;251(3): 470-476.

20. Yu J, Blackford A, Dal Molin M, et al. Time to progression of pancreatic ductal adenocarcinoma from low-to-high tumour stages. Gut. 2015;64(11): 1783-1789.

21. Vasen H, Ibrahim I, Ponce CG, et al. Benefit of surveillance for pancreatic cancer in high-risk individuals: outcome of long-term prospective follow-up studies from three European expert centers. J Clin Oncol. 2016;34(17): 2010-2019.

22. Kardosh A, Lichtensztajn DY, Gubens MA, et al. Long-term survivors of pancreatic cancer: a California population-based study. Pancreas. 2018;47(8): 958-966.

23. Egawa S, Takeda K, Fukuyama S, et al. Clinicopathological aspects of small pancreatic cancer. Pancreas. 2004;28(3):235-240.

24. Jung KW, Kim MH, Lee TY, et al. Clinicopathological aspects of 542 cases of pancreatic cancer: a special emphasis on small pancreatic cancer. J Korean Med Sci. 2007;22(suppl):S79-85

25. Paniccia A, Hosokawa P, Henderson W, et al. Characteristics of 10-year survivors of pancreatic ductal adenocarcinoma. JAMA Surg. 2015;150(8):701-710.

26. Masuda T, Dann AM, Elliott IA, et al. A comprehensive assessment of accurate lymph node staging and preoperative detection in resected pancreatic cancer. J Gastrointest Surg. 2018;22(2):295-302.

27. Tol JA, Gouma DJ, Bassi C, et al. Definition of a standard lymphadenectomy in surgery for pancreatic ductal adenocarcinoma: a consensus statement by the International Study Group on Pancreatic Surgery (ISGPS). Surgery. 2014;156(3): 591-600.

28. Kim HJ, Fay MP, Feuer EJ, et al. Permutation tests for joinpoint regression with applications to cancer rates. Stat Med. 2000;19(3):335-351.

29. Statistical Methodology and Applications Branch, Surveillance Research Program, National Cancer Institute. Joinpoint Regression Program, Version 4.2.0. Bethesda, MD: National Cancer Institute, Statistical Methodology and Applications Branch; 2015.

30. Murphy CC, Singal AG, Baron JA, et al. Decrease in incidence of young-onset colorectal cancer before recent increase. Gastroenterology. 2018;155(6): 1716-1719.e4.

31. Shindo K, Yu J, Suenaga M, et al. Deleterious germline mutations in patients with apparently sporadic pancreatic adenocarcinoma. J Clin Oncol. 2017; 35(30):3382-3390. 
32. Lowery MA, Wong W, Jordan EJ, et al. Prospective evaluation of germline alterations in patients with exocrine pancreatic neoplasms. J Natl Cancer Inst. 2018;110(10):1067-74.

33. Hu C, Hart SN, Polley EC, et al. Association between inherited germline mutations in cancer predisposition genes and risk of pancreatic cancer. JAMA 2018;319(23):2401-2409.

34. Morales-Oyarvide V, Mino-Kenudson M, Ferrone CR, et al. Intraductal papillary mucinous neoplasm of the pancreas in young patients: tumor biology, clinical features, and survival outcomes. J Gastrointest Surg. 2018;22(2): 226-234.

35. He J, Ahuja N, Makary MA, et al. 2564 resected periampullary adenocarcinomas at a single institution: trends over three decades. HPB (Oxford). 2014 16(1):83-90.

36. Jang KM, Kim SH, Kim YK, et al. Missed pancreatic ductal adenocarcinoma: assessment of early imaging findings on prediagnostic magnetic resonance imaging. Eur J Radiol. 2015;84(8):1473-1479.

37. Huang L, Jansen L, Balavarca Y, et al. Resection of pancreatic cancer in Europe and USA: an international large-scale study highlighting large variations. Gut. 2019;68(1):130-139.

38. Sharma A, Kandlakunta H, Nagpal SJS, et al. Model to determine risk of pancreatic cancer in patients with new-onset diabetes. Gastroenterology. 2018; 155(3):730-739.e3.

39. Flaherty S, Mortele KJ, Young GJ. Utilization trends in diagnostic imaging for a commercially insured population: a study of Massachusetts residents 2009 to 2013. J Am Coll Radiol. 2018;15(6):834-841.

40. Horny M, Burgess JF Jr, Cohen AB. Advanced imaging utilization trends in privately insured patients from 2007 to 2013. J Am Coll Radiol. 2015;12(12 pt B): 1380-1387.e4
41. Hamra GB, Semelka RC, Burke LM, et al. Trends in diagnostic CT among feefor-service enrollees, 2000-2011. J Am Coll Radiol. 2014;11(2):125-130.

42. El-Haddad B, Dong F, Kallail KJ, et al. Association of marital status and colorectal cancer screening participation in the USA. Colorectal Dis. 2015;17(5): 0108-14.

43. Jamal A, King BA, Neff LJ, et al. Current cigarette smoking among adults-United States, 2005-2015. MMWR Morb Mortal Wkly Rep. 2016;65(44):1205-1211.

44. Anderson MA, Zolotarevsky E, Cooper KL, et al. Alcohol and tobacco lower the age of presentation in sporadic pancreatic cancer in a dose-dependent manner: a multicenter study. Am J Gastroenterol. 2012;107(11):1730-1739.

45. Niederhuber JE, Brennan MF, Menck HR. The National Cancer Data Base report on pancreatic cancer. Cancer. 1995;76(9):1671-1677.

46. Khawja SN, Mohammed S, Silberfein EJ, et al. Pancreatic cancer disparities in African Americans. Pancreas. 2015;44(4):522-527.

47. Tavakkoli ASA, Waljee A, Elmunzer BJ, et al. Racial disparities and trends in pancreatic cancer incidence and mortality in the United States. Clin Gastroenterol Hepatol. 2020;18:171-178.e10.

48. Jemal A, Lin CC, Davidoff AJ, et al. Changes in insurance coverage and stage at diagnosis among nonelderly patients with cancer after the affordable care act. J Clin Oncol. 2017;35(35):3906-3915.

49. Han X, Yabroff KR, Ward E, et al. Comparison of insurance status and diagnosis stage among patients with newly diagnosed cancer before vs after implementation of the patient protection and affordable care act. JAMA Oncol. 2018;4(12):1713-1720.

50. Duggan MA, Anderson WF, Altekruse S, et al. The Surveillance, Epidemiology, and End Results (SEER) program and pathology: toward strengthening the critical relationship. Am J Surg Pathol. 2016;40(12):e94-e102. 